\title{
Tunneling current spectroscopy of a nanostructure junction involving multiple energy levels
}

\author{
David M.-T. Kuo ${ }^{1}$, Yia-Chung $\mathrm{Chang}^{2,3 *}$ \\ ${ }^{1}$ Department of Electrical Engineering, National Central University, Chung-Li, Taiwan 320 \\ ${ }^{2}$ Research Center for Applied Sciences, Academia Sinica, Taipei, Taiwan 115 \\ ${ }^{3}$ Department of Physics, University of Illinois at Urbana-Champaign, Urbana, Illinois 6180
}

(Dated: November 12, 2017)

\begin{abstract}
A multi-level Anderson model is employed to simulate the system of a nanostructure tunnel junction with any number of one-particle energy levels. The tunneling current, including both shelltunneling and shell-filling cases, is theoretically investigated via the nonequilibrium Green's function method. We obtain a closed form for the spectral function, which is used to analyze the complicated tunneling current spectra of a quantum dot or molecule embedded in a double-barrier junction. We also show that negative differential conductance can be observed in a quantum dot tunnel junction when the Coulomb interactions with neighboring quantum dots are taken into account.
\end{abstract}

In the last decade, the tunneling current spectra of nanostructure junctions such as single-electron transistors (SETs) ${ }^{1-2}$ and molecule transistors $(\mathrm{MTs})^{3-5}$ have been extensively studied due to their important applications in quantum computing, quantum communication, and ultrahigh-density IC circuits. Although the singleparticle energy levels of such systems can be obtained by $a b$ inito or semiempirical methods ${ }^{6-10}$, it is still difficult to model the full tunneling current spectra due to the presence of Coulomb blockade and the uncertainty in dot shape, size and position.

The characteristics of tunneling current of SETs or MTs can be classified into either the shell-tunneling case (with no charge accumulation) or the shell-filling case (with charge accumulation). In the shell-tunneling case, the electron Coulomb interactions are suppressed. Consequently, current spectra directly reveals the oneparticle resonance energy levels. In the shell-filling case, the tunneling current spectra become much more complicated due to the presence of electron Coulomb interactions. Consequently, the charging energies and energy levels can not be readily and directly obtained from experimental data. Both physical parameters are crucial not only in the optimization of tunneling devices, but also for the understanding of fundamental physics of miniaturized nanostructures. Thus, it is desirable to derive a simple formula that allows one to determine the multiple energy levels involved and the associated Coulomb interactions from the measured tunneling current spectra.

In this letter, we derive a simple yet general analytic expression that is applicable for modeling the tunneling current through any nanostructure (including QDs and molecules) involving multiple energy levels (i.e. the ground state plus all the excited states of interest). This is done by solving a multi-level Anderson model via nonequilibrium Green's function technique, ${ }^{11}$ which has been extensively used for investigating the Coulomb blockade and Kondo effect on the tunneling current through the ground state of a single QD. ${ }^{12}$ We find that when more than one level in a $\mathrm{QD} /$ molecule are involved in the charge transport, the average two-particle occupation numbers play an important role in determining whether a given energy channel is close or open to the tunneling current.

The system of an isolated nanostructure embedded in a double-barrier junction can be described by the following Hamiltonian ${ }^{13-16}$ :

$$
\begin{aligned}
H & =\sum_{k, \sigma, \beta} \epsilon_{k} a_{k, \sigma, \beta}^{\dagger} a_{k, \sigma, \beta}+\sum_{\ell, \sigma} E_{\ell} d_{\ell, \sigma}^{\dagger} d_{\ell, \sigma} \\
& +\sum_{\ell, j, \sigma, \sigma^{\prime}} U_{\ell, j} d_{\ell, \sigma}^{\dagger} d_{\ell, \sigma} d_{j, \sigma^{\prime}}^{\dagger} d_{j, \sigma^{\prime}}+\sum_{k, \sigma, \beta, \ell} V_{k, \beta, \ell} a_{k, \sigma, \beta}^{\dagger} d_{\ell, \sigma} \\
& +\sum_{k, \sigma, \beta, \ell} V_{k, \beta, \ell}^{*} d_{\ell, \sigma}^{\dagger} a_{k, \sigma, \beta}
\end{aligned}
$$

where $a_{k, \sigma, \beta}^{\dagger}\left(a_{k, \sigma, \beta}\right)$ creates (destroys) an electron of momentum $k$ and spin $\sigma$ with energy $\epsilon_{k}$ in the $\beta$ metallic electrode. $d_{\ell, \sigma}^{\dagger}\left(d_{\ell, \sigma}\right)$ creates (destroys) an electron inside the nanostructure with orbital energy $E_{\ell} . U_{\ell, j}$ describes the intra- or inter-level charging energies, and $V_{k, \beta, \ell}$ describes the coupling between the band states in the contacts and the confined states in the nanostructure.

Using Keldysh Green's function technique ${ }^{11,12}$, we obtain the tunneling current through a nanostructure,

$$
J=\frac{-2 e}{\hbar} \sum_{\ell} \int \frac{d \epsilon}{2 \pi} \frac{\left(f_{L}-f_{R}\right) \Gamma_{\ell, L}(\epsilon) \Gamma_{\ell, R}(\epsilon)}{\Gamma_{\ell, L}+\Gamma_{\ell, R}} \operatorname{Im} G_{\ell, \sigma}^{r},
$$

where $f_{L}=f\left(\epsilon-\mu_{L}\right)$ and $f_{R}=f\left(\epsilon-\mu_{R}\right)$ are the Fermi distribution functions for the left and right electrode, respectively. $G_{\ell, \sigma}^{r}(\epsilon)$ is the retarded Green's function. The chemical potential difference between these two electrodes is equal to the applied bias $e V_{a} . \Gamma_{\ell, L}$ and $\Gamma_{\ell, R}\left[\Gamma_{\ell, \beta}=\sum_{\mathbf{k}}\left|V_{\ell, \beta, \mathbf{k}}\right|^{2} \delta\left(\epsilon-\epsilon_{\mathbf{k}}\right)\right]$ denote the tunneling rates from the nanostructure to the left (source) and right (drain) electrodes, respectively. For simplicity, these tunneling rates will be assumed to be energy independent. Therefore, the calculation of tunneling current is entirely determined by the spectral function, $A(\epsilon)=\operatorname{Im} G_{\ell, \sigma}^{r}(\epsilon)$.

Rigorous solution to $G_{\ell, \sigma}^{r}$ in the Coulomb blockade regime can be obtained by solving a hierarchy of equations of motion, which relate $G_{\ell, \sigma}^{r}$ to two-particle Green's functions, which can be solved due to the termination of 
the series at some finite order. After tedious algebra, we obtain a neat closed form (by keeping the two-particle correlation functions associate with the same level)

$$
\begin{aligned}
G_{\ell, \sigma}^{r}(\epsilon) & =\left(1-N_{\ell \cdot-\sigma}\right) \sum_{m=1}^{3^{n-1}} \frac{p_{m}}{\epsilon-E_{\ell}-\Pi_{m}+i \frac{\Gamma_{\ell}}{2}} \\
& +N_{\ell \cdot-\sigma} \sum_{m=1}^{3^{n-1}} \frac{p_{m}}{\epsilon-E_{\ell}-U_{\ell}-\Pi_{m}+i \frac{\Gamma_{\ell}}{2}},
\end{aligned}
$$

where $n$ denotes the number of one-particle energy levels considered for the nanostructure. $\Pi_{m}$ denotes the sum of Coulomb interactions seen by a particle in level $\ell$ due to other particles in configuration $m$, in which each level $j(j \neq \ell)$ can be occupied by zero, one or two particles. $p_{m}$ denotes the probability of finding the system in configuration $m$. For a two-level $(n=2)$ system $(\ell \neq j)$, we have three configurations with $p_{1}=a^{j} \equiv 1-\left(N_{j, \sigma}+N_{j,-\sigma}\right)+$ $\left\langle n_{j, \sigma} n_{j,-\sigma}\right\rangle$ (the probability with no particle in level $j$ ), $p_{2}=b^{j} \equiv N_{j, \sigma}+N_{j,-\sigma}-2\left\langle n_{j, \sigma} n_{j,-\sigma}\right\rangle$ (the probability with one particle in level $j$ ), and $p_{3}=c^{j} \equiv\left\langle n_{j, \sigma} n_{j,-\sigma}\right\rangle$ (the probability with two particles in level $j$ ). Meanwhile, $\Pi_{1}=0, \Pi_{2}=U_{\ell j}$ and $\Pi_{3}=2 U_{\ell j} . U_{\ell j}$ denotes the interlevel Coulomb interaction between an electron at level $\ell$ and the other at level $j$. For a three-level system $\left(\ell \neq j \neq j^{\prime}\right)$, there are nine $(3 \times 3)$ configurations, and the probability factors become $p_{1}=a^{j} a^{j^{\prime}}$, $p_{2}=b^{j} a^{j^{\prime}}, p_{3}=a^{j} b^{j^{\prime}}, p_{4}=c^{j} a^{j^{\prime}}, p_{5}=c^{j^{\prime}} a^{j}, p_{6}=b^{j} b^{j^{\prime}}$, $p_{7}=c^{j} b^{j^{\prime}}, p_{8}=c^{j^{\prime}} b^{j}$, and $p_{9}=c^{j} c^{j^{\prime}}$. Interlevel Coulomb interaction factors are $\Pi_{1}=0, \Pi_{2}=U_{\ell j}, \Pi_{3}=U_{\ell j^{\prime}}$, $\Pi_{4}=2 U_{\ell j}, \Pi_{5}=2 U_{\ell j^{\prime}}, \Pi_{6}=U_{\ell j}+U_{\ell j^{\prime}}, \Pi_{7}=2 U_{\ell j}+U_{\ell j^{\prime}}$, $\Pi_{8}=2 U_{\ell j^{\prime}}+U_{\ell j}$, and $\Pi_{9}=2 U_{\ell j}+2 U_{\ell j^{\prime}}$. Based on these simple rules, the probability factors and interlevel Coulomb interaction factors for any number of energy levels can be similarly determined. We see that the probability of finding the system in each configuration is determined not only by the average one-particle occupation numbers but also by the average two-particle occupation numbers. $N_{\ell, \sigma}$ in Eq. (3) can be obtained by solving the following equations self-consistently

$$
\begin{gathered}
N_{\ell, \sigma}=-\int \frac{d \epsilon}{\pi} \frac{\Gamma_{\ell, L} f_{L}+\Gamma_{\ell, R} f_{R}}{\Gamma_{\ell, L}+\Gamma_{\ell, R}} \operatorname{Im} G_{\ell, \sigma}^{r}(\epsilon), \\
\left\langle n_{\ell, \sigma} n_{\ell,-\sigma}\right\rangle=-\int \frac{d \epsilon}{\pi} \frac{\Gamma_{\ell, L} f_{L}+\Gamma_{\ell, R} f_{R}}{\Gamma_{\ell, L}+\Gamma_{\ell, R}} \operatorname{Im}_{\ell, \ell}^{r}(\epsilon), \\
G_{\ell, \ell}^{r}(\epsilon)=N_{\ell \cdot-\sigma} \sum_{m=1}^{3^{n-1}} \frac{p_{m}}{\epsilon-E_{\ell}-U_{\ell}-\Pi_{m}+i \frac{\Gamma_{\ell}}{2}} .
\end{gathered}
$$

Both $N_{\ell, \sigma}$ and $N_{\ell, \ell} \equiv\left\langle n_{\ell, \sigma} n_{\ell,-\sigma}\right\rangle$ are limited to the range between 0 and 1 .

To illustrate the usefulness of our theory, we calculate the tunneling current spectra of an isolated CdSe QD sandwiched between an STM tip (left lead) and a conducting substrate (right lead) as studied experimentally in Ref. 17. We consider the case of three energy levels. Because the tip-substrate is biased at $V_{a}$, the bare energy levels of $E_{\ell}$ in the dot are changed to $E_{\ell}+\alpha e V_{a}$. The factor $\alpha=L_{L} /\left(L_{L}+L_{R}\right)$ is determined by the distances from the QD center to the tip $\left(L_{L}\right)$ and the substrate $\left(L_{R}\right)$, respectively. Here $\alpha=0.61$, which is determined from the separation between the first peak at negative bias and that at positive bias $\frac{17}{7}$ Other physical parameters can also be determined by comparing Eq. (3) with the peak positions of the tunneling spectra observed in Ref. 17. We obtain the following. The chemical potentials of both electrodes are $0.78 \mathrm{eV}$ below the ground state level $E_{1}$ at zero bias. $E_{2}-E_{1}=0.236 \mathrm{eV}, E_{3}-E_{1}=0.456 \mathrm{eV}, U_{1}=0.137 \mathrm{eV}$, $U_{12}=U_{21}=0.122 \mathrm{eV}, U_{2}=0.07 \mathrm{eV}, U_{3}=0.06 \mathrm{eV}$, $U_{13}=U_{31}=0.1 \mathrm{eV}$, and $U_{23}=U_{32}=0.04 \mathrm{eV}$. These intra- and inter-level Coulomb energies are reasonable for the $3 \mathrm{~nm}$ diameter CdSe QD considered here. We note that the p-like level in a spherical QD is sixfold degenerate (including spin). However, the coupling strength between the tip (or substrate) and the $p_{x^{-}}$or $p_{y}$-like orbital is weak. Therefore, only $p_{z}$-like orbital has been included, which is labeled by $E_{2}$, while $E_{3}$ denotes one of the d-like orbitals that is strongly coupled to the leads.

Occupation numbers $N_{\ell}$ and $N_{\ell, \ell}$ are obtained by solving the coupled equations Eqs. (4)-(6). Once they are determined, we can calculate the tunneling current by substituting Eq. (3) into Eq. (2). Figure 1 shows the differential conductance, $d J / d V_{a}$ as a function of applied bias for various ratios of $\Gamma_{L} / \Gamma_{R}$. Curve (c) exhibits the maximum number of resonant channels, which correspond to the poles of the retarded Green's function in Eq. (3). The first eight peaks of curve(c) (with energy less than $E_{3}$ ) are $\epsilon_{1}=E_{1}, \epsilon_{2}=E_{1}+U_{1}, \epsilon_{3}=E_{2}, \epsilon_{4}=E_{1}+U_{1}+U_{12}, \epsilon_{5}=$ $E_{2}+U_{2}, \epsilon_{6}=E_{2}+U_{12}, \epsilon_{7}=E_{1}+U_{1}+2 U_{12}$, and $\epsilon_{8}=E_{2}+U_{2}+U_{12}$. The charging energy of the ground state, $U_{1}$ can be determined by the difference in bias between the first two peaks. The two channels, $\epsilon=E_{1}+U_{12}$ and $\epsilon=E_{1}+2 U_{12}$ are prohibited because those resonance energies are below $E_{2}$ (hence $N_{2}=0$ and $N_{22}=0$ when the applied bias aligns with these channels). The limit $\Gamma_{L} / \Gamma_{R} \ll 1$ corresponds to the so called "shell-tunneling case". ${ }^{17}$ According to Eqs. (4) and (5), $N_{\ell}$ and $N_{\ell, \ell}$ are determined by the factor $\mathcal{P}=\Gamma_{\ell, L} /\left(\Gamma_{\ell, L}+\Gamma_{\ell, R}\right)$. Therefore, $N_{\ell}$ and $N_{\ell, \ell}$ approaches zero in this limit. Consequently, the peaks associated with the resonance levels involving the intralevel and interlevel Coulomb interactions will be suppressed, since the carrier will tunnel out much faster than it can be fed. The peaks associated with the single-particle levels, $E_{1}, E_{2}$ and $E_{3}$ will be the only resonance channels allowed in the limit of $\Gamma_{L} / \Gamma_{R} \ll 1$ as seen in curve (e). In the opposite limit, $\Gamma_{L} / \Gamma_{R} \gg 1$ we have the so called "shell-filling case" [see curve(a)]. In this case, both $N_{1}$ and $N_{11}$ approach 1 when $V_{a}>E_{1}+U_{1}$. Consequently, resonance channels related at $E_{2}$ and $E_{2}+U_{2}$ are suppressed while the resonances at $E_{2}+2 U_{12}$ and $E_{2}+U_{2}+2 U_{12}$ are enhanced. This indicates that the bare energy levels are renormalized if charges reside in the QD. Similarly for $E_{3}$ related channels. 
Next, we compare our theoretical prediction for the tunneling current spectra with those measured in Ref.17, where the observed differential conductance peaks are broadened. The broadening is mainly due to coupling to nearby QDs since the experiment was performed at $T=4 K$. To take into account the above broadening effect, we replace each Lorentzian function appearing in the differential conductance by a Gaussian function of the form $f_{i} \exp \left\{-\frac{\left(\epsilon-\epsilon_{i}\right)^{2}}{2 \rho_{i}^{2}}\right\} /\left(\rho_{i} \sqrt{2 \pi}\right) . \quad f_{i}, \epsilon_{i}$ and $\rho_{i}$ denote the peak strength, resonance energy, and broadening width, respectively. Figure 2 shows the predicted differential conductance as a function of applied bias at zero temperature. We have used the following parameters $\Gamma_{L, 1}=1 \mathrm{meV}, \Gamma_{R, 1}=0.15 \mathrm{meV}, \Gamma_{L, 2}=3 \mathrm{meV}$, $\Gamma_{R, 2}=0.6 \mathrm{meV} \cdot \Gamma_{L, 3}=1.5 \mathrm{meV}, \Gamma_{R, 3}=0.375 \mathrm{meV}$. The variation in tunneling rates for different levels reflects the difference in wave functions and the bias-dependent barrier height. For simplicity, we assume $\rho_{i}=\rho+\Gamma_{i}$, where $\rho=35 \mathrm{meV}$ characterizes the broadening due to coupling to nearby QDs. Both the positions and relative strengths of these peaks are in very good agreement with the experimental measurement reported in Ref. 17. If we remove the extrinsic broadening by setting $\rho=0$, the differential conductance spectrum exhibits more resonance channels as shown in the lower part of the figure, which can not be resolved experimentally.

To illustrate the significance of the two-particle occupation number, we apply our theory to the case of a coupled dot embedded between two leads, where two QDs (dot A and $\operatorname{dot} \mathrm{B})$ are coupled by interdot Coulomb interactions, even though the inter-dot tunneling is weak. It is assumed that the right lead is closer to dot A than to $\operatorname{dot} \mathrm{B}$. Thus, the tunneling rate $\Gamma_{R, B}$ is ten times smaller than $\Gamma_{R, A}$, and the total current is dominated by the current through dot $\mathrm{A}\left(J_{A}\right)$. Figure 3 shows the total tunneling current, $J=J_{A}+J_{B}$ for various strengths of the interdot Coulomb interaction (which may, for example, correspond to different separations between the two dots). It is seen that negative differential conductance (NDC) can occur due to the interdot Coulomb interaction. The NDC characteristic of tunneling current can be understood mainly from the feature of $J_{A}$, since $J_{A} \gg J_{B}$. NDC first occurs at $E_{B}$ as dot B becomes filled, which leads to a suppression of the current through the channel $E_{A}$, and the current resumes as the channel $E_{A}+U_{A B}$ opens up when the bias further increases. For comparison, we also plot in Fig. 3 the total current, $J_{a}+J_{b}$ obtained with the approximation, $\left\langle n_{j, \sigma} n_{j,-\sigma}\right\rangle=N_{j, \sigma} N_{j,-\sigma}$ (dashdotted curve). The difference between this curve and the solid curve shows the significance of treating the twoparticle occupation number correctly. In particular, we see in the dash-dotted curve $\left(J_{a}+J_{b}\right)$ that the resonance channel at $\epsilon=E_{A}+2 U_{A B}$ is allowed even though the applied bias is below $E_{B}+U_{B}$ (i.e. dot B remains singly charged). Such a nonphysical behavior in $J_{a}+J_{b}$ arises due to the approximation $N_{B B} \approx N_{B} N_{B} \neq 0$ used, while if treated correctly $N_{B B}$ should vanish under this condition. We also notice that the tunneling current increases only slightly when applied bias overcomes the charging energy of dot A (marked by $E_{A}+U_{A}$ ) since we are considering a shell-filling case for $\operatorname{dot} \mathrm{B}\left(\Gamma_{L, B} / \Gamma_{R, B}=10\right)$ here $\left(N_{B} \approx 1\right)$. Increasing the applied bias beyond the resonance channel at $E_{A}+U_{A}+U_{A B}$ leads to a significant jump in the tunneling current. However, this tunneling current is suppressed again at $E_{B}+U_{B}+U_{A B}$ because $\operatorname{dot} \mathrm{B}$ is now filled with two charges.

To further analyze the NDC behavior due to interdot Coulomb interaction, we plot in Figure 4 the differential conductances for various ratios of $\Gamma_{L, B} / \Gamma_{R, B}$. Curve (a) corresponds to the derivative of the curve shown in Fig. 3. The NDC behavior is quite apparent. However, the NDC behavior disappears in curves (b) and (c) when $\operatorname{dot} \mathrm{B}$ is not under the shell-filling condition, even though the interdot Coulomb energy remains the same. When dot B is in the shell tunneling limit $\left(\Gamma_{L, B} \ll \Gamma_{R, B}\right), J_{A}$ becomes unaffected by $\operatorname{dot} \mathrm{B}$, and the tunneling current [curve (c)] exhibits mainly two resonance channels at $E_{A}$ and $E_{A}+U_{A}$ plus a weaker peak at $E_{B}+U_{A B}$, which is contributed from $J_{B}$. As illustrated in Figs. 3 and 4, when one employs an SET as a charge detector, the interdot Coulomb interactions due to neighboring QDs and the tunneling rate ratios, $\Gamma_{L} / \Gamma_{R}$ should be carefully considered in the design.

In conclusion, a closed form expression for the spectral function for nanostructure tunnel junctions involving multiple energy levels has been derived and it has be employed to analyze the complicated spectra of the tunneling current through a realistic single QD. Our present theory also provides a useful guideline for the design of SET with coupled quantum dots. We show that when the interdot hoping effect is weak but the interdot Coulomb interactions are strong, NDC behavior can occur and it depends sensitively on the tunneling rate ratios for incoming and outgoing electrons in each QD.

This work was supported by the National Science Council of the Republic of China under Contract No. NSC-95-2221-E-008-147 and by the Academia Sinica.
* Electronic address: ychang15@uiuc.edu

1 L. Guo, E. Leobanding and S. Y. Chou, Science 275, 64 (1997).

${ }^{2}$ H. Ishikuro and T. Hiramoto, Appl. Phys. Lett. 71, 3691 (1997).
${ }^{3}$ M. A. Reed, C. Zhou, C. J. Muller, T. P. Burgin, J. M.

Tour, Science 278, 252 (1997), and references therein.

4 C. Kergueris et al, Phys. Rev. B 59. 12505 (1999).

${ }^{5}$ C. Joachim, J. K. Gimzewski and A. Aviram, Nature. 408, 541 (2000), and references therein. 
${ }^{6}$ S. Kubatkin et al, Nature 425. 698 (2003).

7 H. park, J. Park, A. K. L. Lim, E. H. Anderson. A. P. Alivisatos and P. L. Mceuen, Nature, 407. 57 (2000).

8 C. C. Kaun and T. Seideman, Phys. Rev. lett. 94. 226801 (2005).

9 O. Stier, M. Grundmann, and D. Bimberg, Phys. Rev. B 59, 5688 (1999).

10 S. J. Sun and Y. C. Chang Phys. Rev. B 62, 13631 (2000).

11 L. V. Keldysh: Zh. Eksp, Teor. Fiz. 471515 (1964) [Sov. Phys. JETP 201018 (1965)].

12 A. P. Jauho, N. S. Wingreen, and Y. Meir: Phys. Rev. B 505528 (1994), and references therein.

13 U. Banin, Y. Cao, D. Katz, O. Millo, Nature 400, 542(1999).

14 O. Millo, D. Katz, Y. W. Cao and U. Banin, Phys. Rev. Lett. 865751 (2001).

15 Y. M. Niquet, C. Delerue, G. Allen, and M. Lannoo, Phys. Rev. B 65, 165334 (2002).

16 S. W. Wu, G. V. Nazin, X. Chen, X. H. Qiu and W. Ho, Phys. Rev. Lett. 93, 236802 (2004).

17 L. Jdira, P. Liljeroth, E. Stoffels, D. Vanmaekelbergh, and S. Speller, Phys. Rev. B 73, 115305 (2006).
Figure captions

Fig. 1 Differential conductance as a function of applied bias at for various ratios of $\Gamma_{L} / \Gamma_{R}$ : (a) 10 , (b) 5 , (c) 1 , (d) 0.4 , and (e) $0.2 . \Gamma_{R}$ is fixed at $1 \mathrm{meV}$.

Fig. 2 Differential conductance as a function of applied bias with and without the extrinsic broadening effect due to coupling to nearby QDs. For better display, the broadened spectra has been multiplied by a factor 5 .

Fig. 3 Tunneling current $\left(J_{A}+J_{B}\right)$ as a function of bias for various strengths of interdot Coulomb interaction, $U_{A B}$. The parameters used are: $E_{A}=E_{F}+108 \mathrm{meV}$, $E_{B}=E_{A}+10 \mathrm{meV}, U_{A}=111 \mathrm{meV}, U_{B}=108 \mathrm{meV}$, $\Gamma_{L, A}=\Gamma_{R, A}=\Gamma_{L, B}=1 \mathrm{meV}$, and $\Gamma_{R, B}=0.1 \mathrm{meV}$, and $\alpha=0.61$. Current is in units of $J_{0}=\mathrm{e}^{*}(\mathrm{meV}) / \mathrm{h}$. Dot-dashed curve is for $J_{a}+J_{b}$ with $U_{A B}=30 \mathrm{meV}$.

Fig. 4 Differential conductance as a function of applied bias for various tunneling rate ratios of $\Gamma_{L, B} / \Gamma_{R, B}$. $U_{A B}=30 \mathrm{meV}$. (a) $\Gamma_{L, B}=1 \mathrm{meV}$ and $\Gamma_{R, B}=0.1 \mathrm{meV}$. (b) $\Gamma_{L, B}=1 \mathrm{meV}$ and $\Gamma_{R, B}=1 \mathrm{meV}$. (c) $\Gamma_{L, B}=$ $0.1 \mathrm{meV}$ and $\Gamma_{R, B}=1 \mathrm{meV}$. 


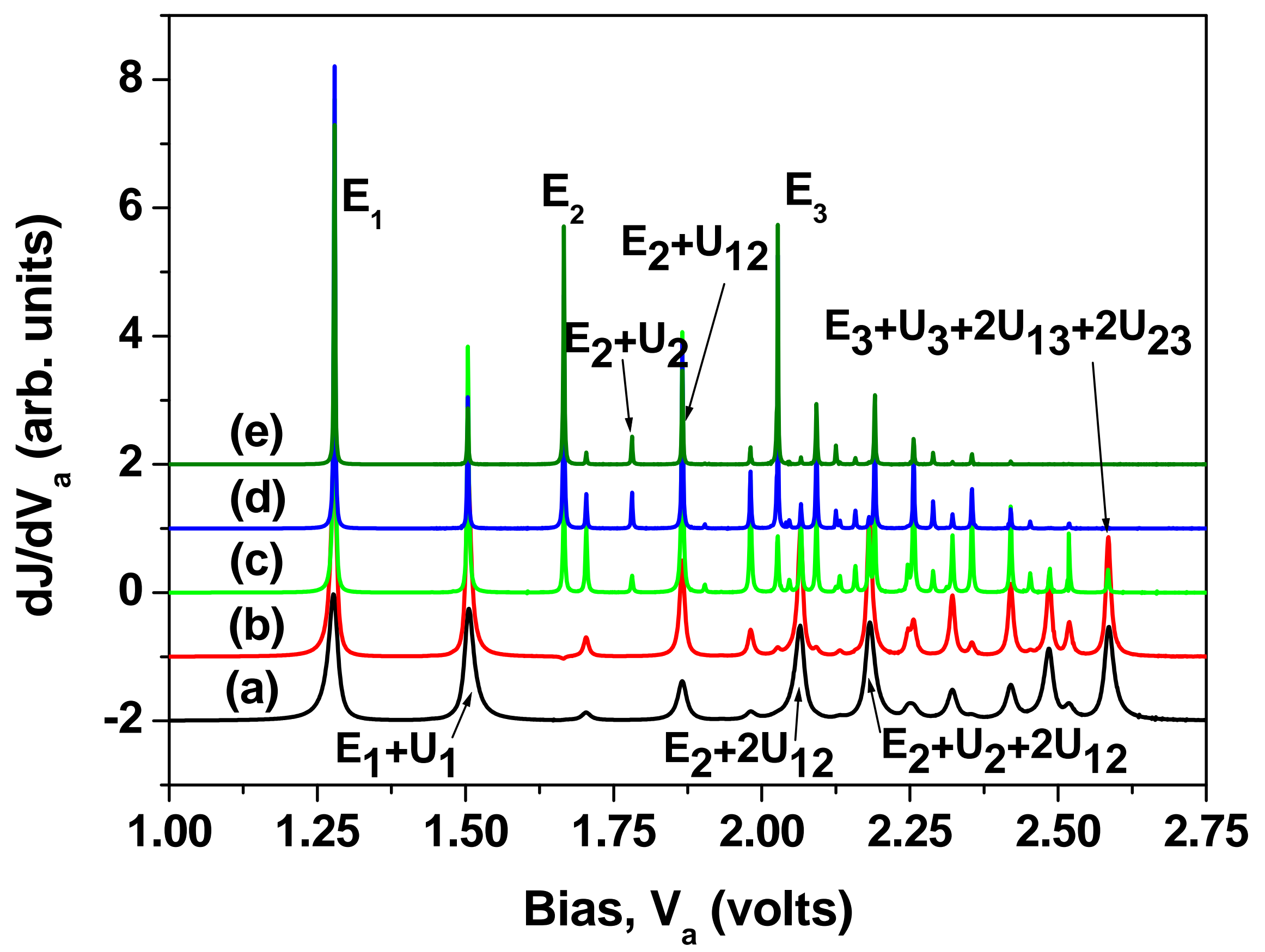




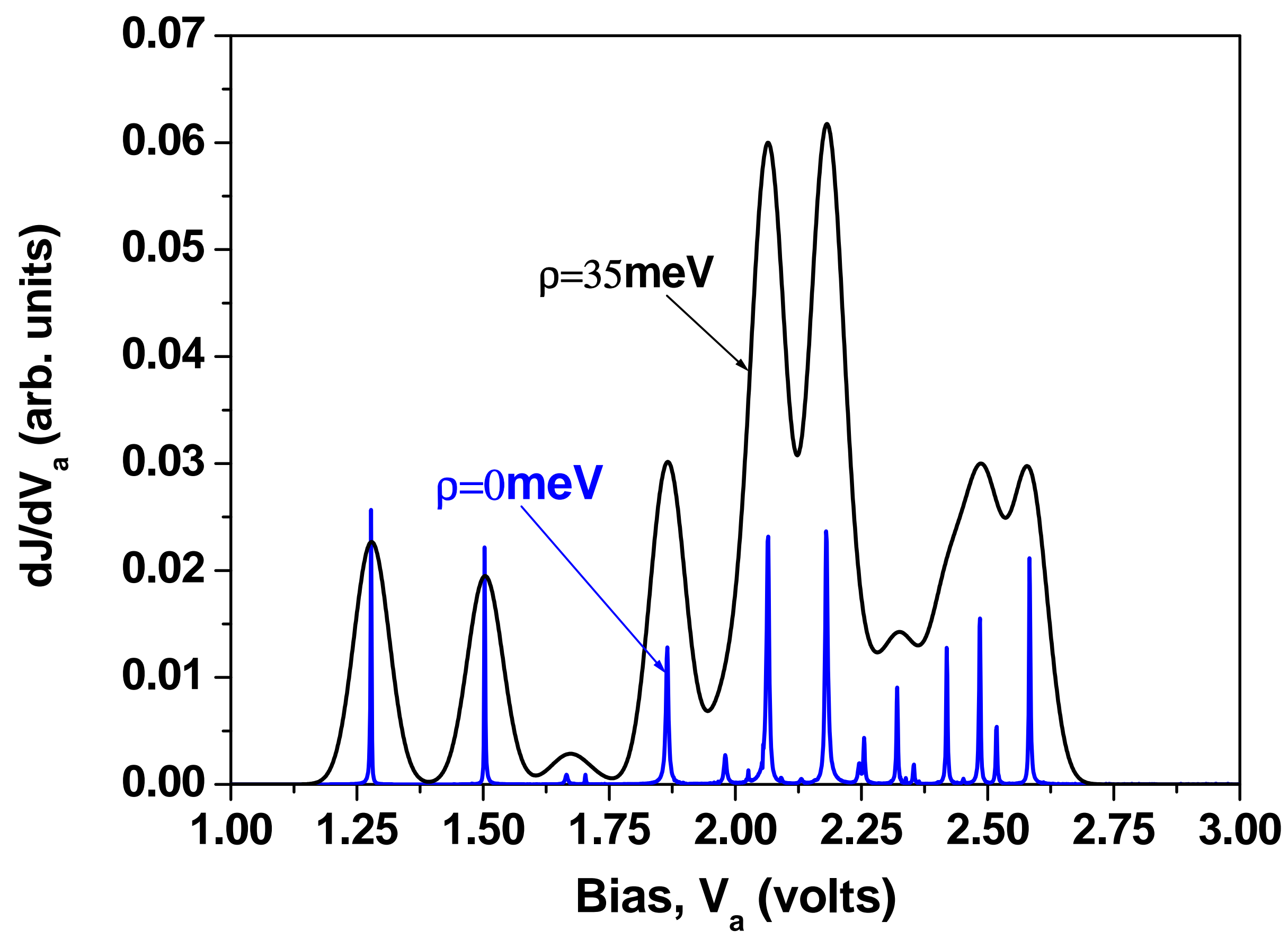




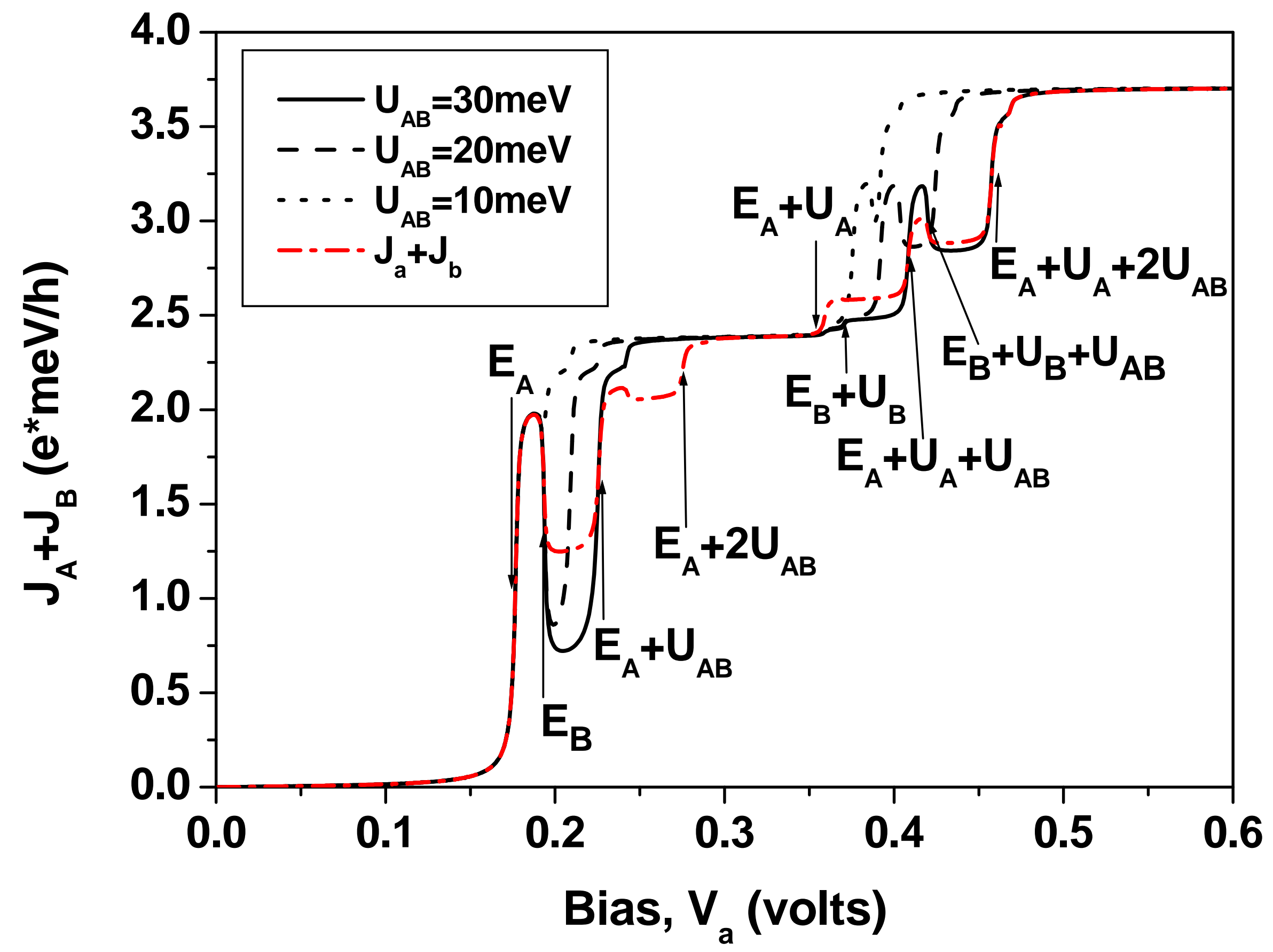




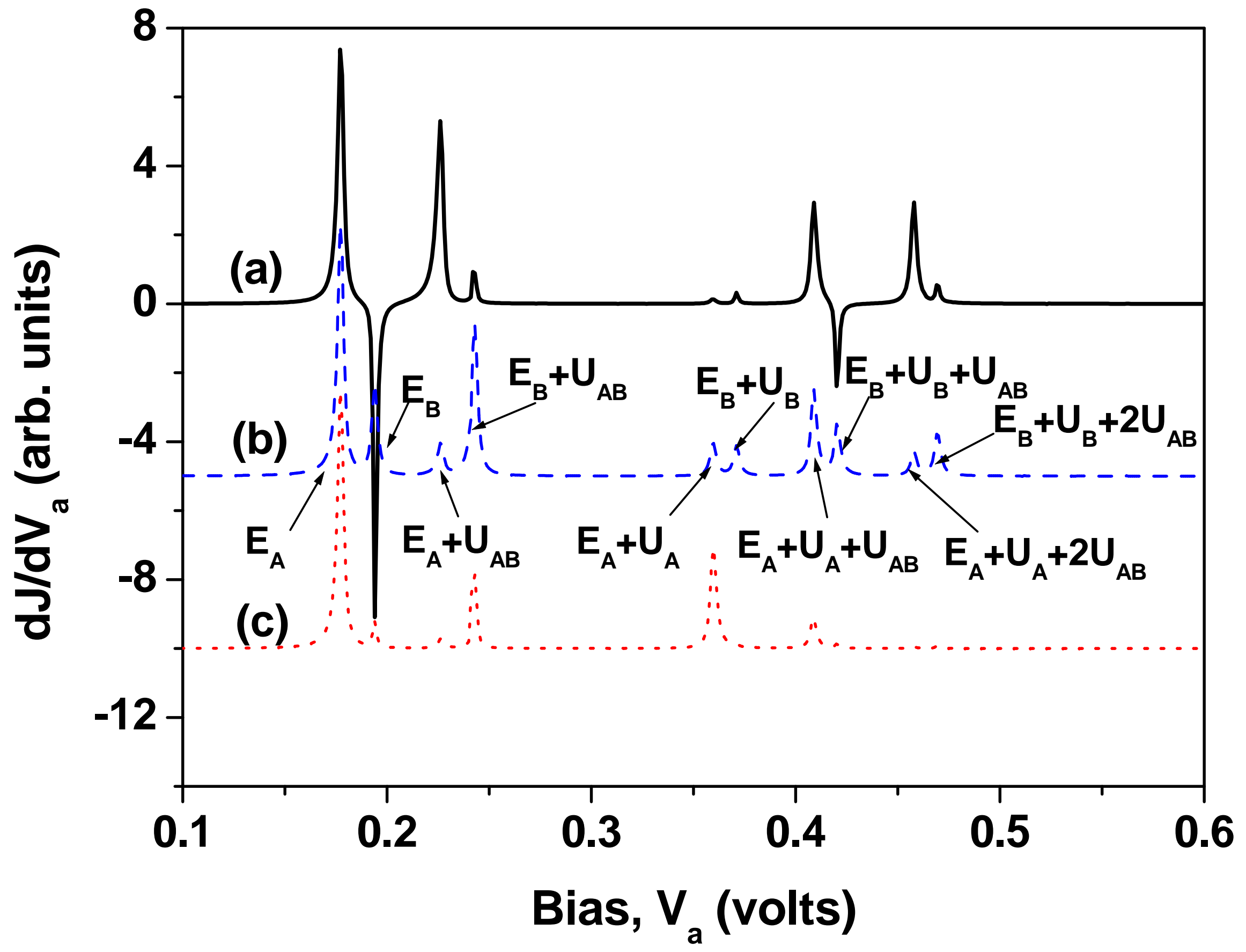

